\title{
Towards Quantitative EDX Results in 3 Dimensions
}

\author{
Bart Goris ${ }^{1}$, Bert Freitag ${ }^{2}$, Daniele Zanaga ${ }^{1}$, Eva Bladt ${ }^{1}$, Thomas Altantzis ${ }^{1}$, Jan Ringnalda ${ }^{2}$, and Sara \\ Bals ${ }^{1}$
}

1. EMAT, University of Antwerp, Antwerp, Belgium

2. FEI Company, P.O. Box 80066, KA 5600 Eindhoven, The Netherlands

Electron tomography has become a versatile tool to investigate a broad variety of (hetero)nanostructures in 3 dimensions (3D) [1]. Recent developments even pushed the resolution of the technique to the atomic level [2-4]. Nevertheless, it remains challenging to resolve the chemical composition of complex nanostructures in $3 \mathrm{D}$.

In principle, energy dispersive X-ray (EDX) mapping can be combined with electron tomography since the number of generated X-rays scales linearly with sample thickness. However, early attempts to perform 3D EDX experiments were complicated by the specimen-detector geometry [5]. Therefore, recent efforts lead to a novel design of the EDX detector system, which enables one to apply 3D EDX mapping to different structures [6]. An example of a 3D EDX reconstruction, obtained using 2D EDX maps that have been acquired using a probe corrected Titan, equipped with a Super-X system is presented in Figure 1. The reconstruction shows a Au@Ag nanocube of which the Au core yields an octahedral shape [7]. This example clearly illustrates the potential of 3D EDX mapping, but one needs to be careful when extracting quantitative information from such reconstructions. To reach this goal, the different steps of an EDX tomography experiment need to be optimized.

The Super-X system consists of 4 EDX detectors symmetrically arranged with respect to the sample. To obtain a reliable $3 \mathrm{D}$ reconstruction, we quantified the counts detected by each of these detectors as a function of tilt angle. This is of great importance since shadowing effects due to the holder might occur. Figure 2 presents the EDX counts that were acquired from a spherical Au particle using a Model 2030 Fischione tomography holder. Due to the symmetry of the sample, the collected signal should in principle be independent of the tilt angle. Using this dedicated holder, shadowing is kept at a strict minimum, but even in this optimal case, a tilt dependent collection efficiency of the detector is observed. This problem, caused by remaining shadowing of the sample grid, can be overcome by combining EDX signals, unaffected by shadowing, that are collected by different detectors during the tilt series.

Quantification of the EDX maps is the next crucial step. Often, EDX quantification is performed using the "Cliff-Lorimer" method, originally developed for the investigation of thin foils [8]. However, when a sample is tilted, the projected thickness might vary, which will hamper a straightforward quantitative $3 \mathrm{D}$ reconstruction. Here, we will evaluate the use of the " $Z$ (zeta)-factor" method for quantitative 3D EDX reconstructions by using the following equation [9]:

$$
\rho t=\zeta \frac{1}{C D_{e}}
$$


In this formula, $\mathrm{p}$ is the density of the material and $\mathrm{t}$ equals sample thickness, which we will measure from 3D high angle annular dark field STEM (HAADF-STEM) reconstructions. For monometallic nanostructures, the concentration $\mathrm{C}$ equals 1 . By measuring the intensity $\mathrm{I}$ and the electron dose, the $\zeta$-factor can be determined. After estimation of the Z-factor for different elements, quantitative 3D elemental analysis becomes possible for heteronanomaterials that have unknown composition [10].

\section{References:}

[1] PA Midgley, RE Dunin-Borkowski, Nature Materials 8 (2009), p. 271

[2] S Van Aert et al, Nature 470 (2011), p. 374

[3] MC Scott et al, Nature 483 (2012), p. 444

[4] B Goris et al, Nature Materials 11 (2012), p. 930

[5] G Möbus, RC Doole and BJ Inkson, Ultramicroscopy 96 (2003), p. 433

[6] P Schlossmacher et al, Microscopy Today 18 (2010), p. 14

[7] S Gómez-Graña et al, The journal of physical chemistry letters 4 (2013), p. 2209

[8] G Cliff and GW Lorimer, Journal of Microscopy 103 (1975), p. 203

[9] M Watanabe and DB Williams, Journal of Microscopy 221 (2006) p. 89

[10] The authors acknowledge financial support from European Research Council (ERC Starting Grant \#335078-COLOURATOMS). The work was supported by the Flemish Fund for Scientific Research (FWO Vlaanderen) through PhD research grant to B.G and E.B. The authors would like to thank Prof. L. Liz-Marzán for the provision of the samples and fruitful discussions.

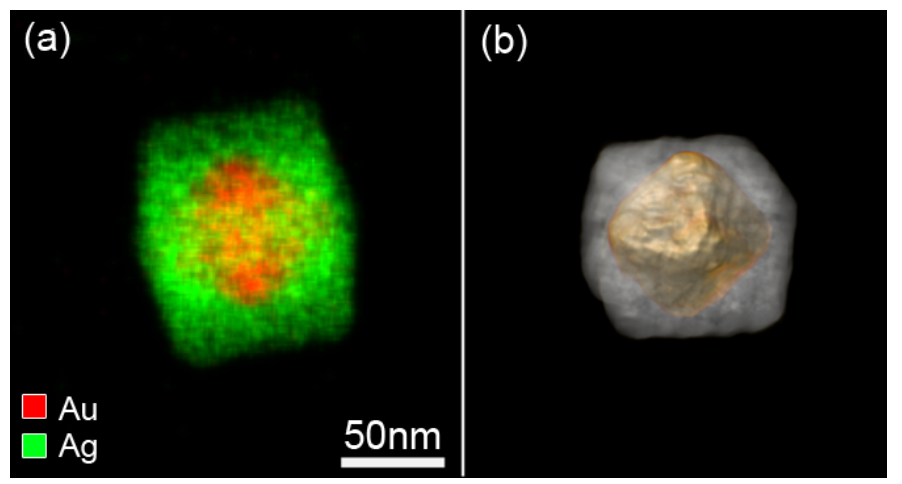

Figure 1: (a) 2D EDX map of a Au@Ag nanocube. Based on a tilt series of such 2D EDX maps, 3D reconstructions (b) could be obtained showing the 3D distribution of the different chemical elements.

(a)

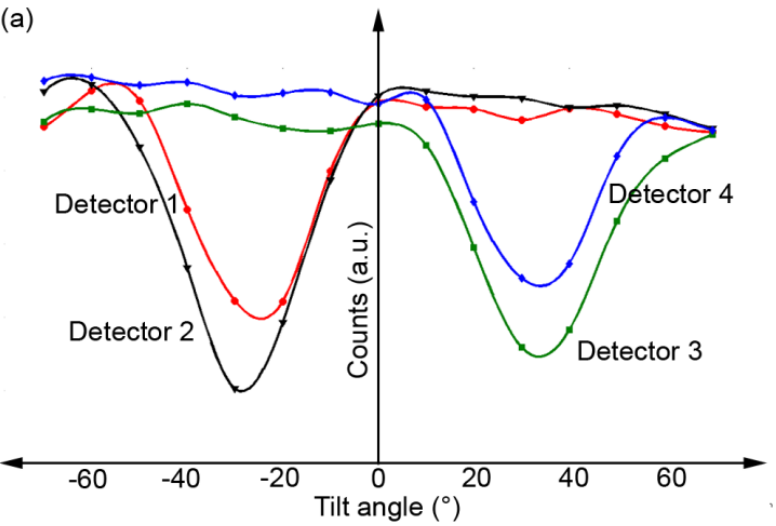

(b)

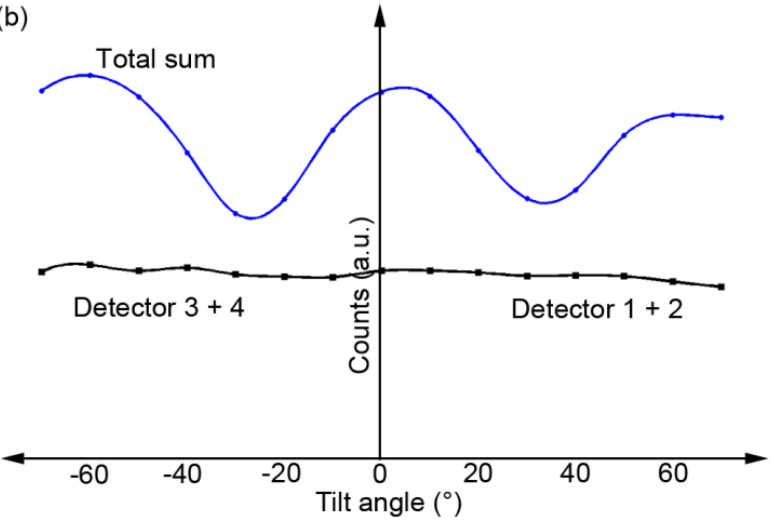

Figure 2: (a) Detected X-ray counts as function of tilt angle for each individual detector of the Super-X system. At certain tilt angles, shadowing effects may block the X-rays preventing them to reach the detectors. (b) Total X-ray count when adding the signal from different detectors. When combining the signals from different detectors in an optimized manner, the resulting signal is independent of tilt angle. 